\title{
Antimicrobial Activity of Cold Plasma Treatment on Acrylic Denture Bases: An In Vitro Evaluation
}

\author{
Mohammad Asnaashari ${ }^{1}$, Salar Motamedi ${ }^{2}$, Negin Asnaashari ${ }^{1}$, Saranaz Azari-Marhabi ${ }^{*}$ \\ ${ }^{1}$ Laser Application in Medical Science Research Center, Shahid Beheshti University of Medical Sciences, Tehran, Iran \\ ${ }^{2}$ Dental Research Center, Research Institute of Dental Sciences, School of Dentistry, Shahid Beheshti University of Medical \\ Sciences, Tehran, Iran
}

\author{
*Correspondence to \\ Saranaz Azari-Marhabi, \\ Laser Application in Medical \\ Science Research Center \\ Shohada Tajrish Hospital, Tajrish \\ Square, Tajrish Street, Tehran, Iran \\ Tel: +98-912-2172508; \\ Fax: +98-21-22749221; \\ Email: dr.s.azari@sbmu.ac.ir
}

Published online December 1, 2019

\begin{abstract}
Introduction: Cross-contamination between dental office and prosthetic laboratories is of utmost importance. The dental prosthesis could harbor tones of microorganisms harmful to health staff. It has been estimated that more than $60 \%$ of the prostheses delivered to clinics from laboratories or vice versa are contaminated with pathogenic microorganisms. Several disinfection methods and chemicals have been proposed for sanitizing prostheses. Cold plasma, or nonthermal plasma, has been introduced as a new method in medical equipment disinfection. The current study aimed to compare the antimicrobial effect of cold plasma and the routinely used alcohol-based impression disinfectant ASEPTOPRINT ${ }^{\circledR}$ on disinfecting dentures.

Methods: In this experimental study, 30 maxillary complete dentures were used as the microbial source. Dentures were randomly divided into 3 groups: (1) cold plasma treatment for 30 seconds, (2) cold plasma for 60 seconds, (3) ASEPTOPRINT ${ }^{\circledR}$ spray. Microbial culturing was taken before and after disinfection in each group on both Blood Agar plates and Soborou Dextrose Agar (SDA) plates. After 48 hours incubation at $37^{\circ} \mathrm{C}$, the number of colonies was counted using a digital colony counter and differences between the groups were analyzed.

Results: A significant reduction in microbial colonies was observed after using all disinfection methods. ASEPTOPRINT ${ }^{\circledR}$ spray showed more antimicrobial effects comparing to cold plasma.

Conclusion: The antimicrobial effect of ASEPTOPRINT ${ }^{\circledR}$ spray solution is more than cold plasma application on both microbial and fungal microorganisms. The time of plasma application was not accompanied by any significant differences in the results.

Keywords: Acrylic dentures; Disinfection; Cold plasma; ASEPTOPRINT ${ }^{\circ}$ spray.
\end{abstract}

\section{Introduction}

Health care staff is endangered by various microorganisms due to cross-contamination in dental practice; therefore, infection control is a pivotal issue in dental procedures. ${ }^{1}$

The transmission of infection between dentists, laboratory technicians, and patients is possible when prosthetic appliances like complete dentures are fabricated, repaired, and adjusted during dental practice if appropriate disinfection is not achieved. The potential risk of contamination with the aerosols and pathogens exists during all dental procedures encompassing trimming, finishing, and polishing, so there is a need for appropriate disinfection techniques which act efficiently on a broad spectrum of microorganisms and are cost-effective, rapid and safe for human's health..$^{2-5}$ The disinfection of the dental impressions is strongly recommended by the health policymakers such as the Centre for Disease Control (CDC), the American Dental
Association (ADA) and the British Dental Association (BDA) to prevent the possible transmission of infectious diseases and cross-contamination. ${ }^{6-8}$ Although guidelines for sterilization and disinfection have been established by these organizations, investigations have shown that proper practice is not always followed. ${ }^{5}$

Nowadays immersion in chemical disinfection agents has been mainly suggested for disinfecting dental prostheses like acrylic resin dentures. These disinfectants include a wide variety of chemicals such as $2 \%$ alkaline glutaraldehyde, $0.5 \%$ and $1 \%$ sodium hypochlorite, $3 \%$ aqueous formaldehyde, hydrogen peroxide, $1 \%$ sodium hypochlorite, $4 \%$ chlorhexidine gluconate, and $3.78 \%$ sodium perborate. $^{9-11}$ The effective elimination of pathogens harbored in the inner and outer surfaces of acrylic dentures is among the advantages of this method. Although affecting physical properties such as transverse strength, hardness, surface decay, and 
roughness of denture base, resins have been postulated as the disadvantages of chemical agents. ${ }^{12-15}$

Disinfectants also may have disadvantages like denture staining and irritative reactions of patients' oral mucosa. It has been reported that some disinfectants such as sodium hypochlorite and glutaraldehyde indicate severe and medium cytotoxicity. ${ }^{16}$ Also, some disinfectants can cause corrosion and tarnishing of denture metal ingredients. To overcome these issues, many alternative disinfection methods have been proposed. . $^{-15}$

During the past two decades, cold plasma has been introduced as a new approach which has increasingly attracted researchers' attention due to its antimicrobial effects against pathogens. ${ }^{17}$ It has also been used for food preservation in the food industry due to its antimicrobial properties. ${ }^{18,19}$ Cold plasma is an ionized gas that generates free electrons, ions, ultraviolet photons, and free radical species which directly contact with the bacterial cell. This radiation can inactivate and kill microorganisms by damaging their DNA with its ultraviolet irradiation and free radicals. ${ }^{20}$ Low-pressure plasma treatment can be an environmentally friendly approach. ${ }^{21}$ The current study aimed to compare the antimicrobial effects of cold plasma and routinely used alcohol-based disinfectant spray (ASEPTOPRINT ${ }^{\circledR}$ ) to find a better way for disinfecting dentures.

\section{Methods}

Sample Collection

In this experimental study, in order to mimic the microbial environment of an oral cavity, 30 maxillary complete dentures were used as the microbial source. After obtaining the informed consent of each patient, all dentures were referred to the prosthetic laboratory of the Dental School, Shahid Beheshti University of Medical Sciences for reline, repair or exchange.

The groups were divided as follows:

1. Disinfected by ASEPTOPRINT ${ }^{\circledR}$ spray according to the manufacturer manual (OCC, Switzerland) (Table 1).

2. Treated by cold plasma treatment for 30 seconds.

3. Treated by cold plasma treatment for 60 seconds.

Each of the maxillary complete dentures was removed and placed on a sterile Petri dish $(20 \times 100 \mathrm{~mm})$ by the patient. Then, for microbial investigations, swab samples were collected and deposited on tubes containing 10 $\mathrm{mL}$ normal saline. Then, the solution was diluted by adding $90 \mathrm{~mL}$ of normal saline and 10 lambdas of this dilution were spread with the spread-plate technique for each sample. In order to complete the investigation of bacteria and fungi which might exist on dentures, SABOURAUD 4\% dextrose agar (SDA) (Merck 1.05438, Darmstadt, Germany) and blood agar (Merck, Darmstadt, Germany) cultures were used in this study. The culture mediums were incubated at $37^{\circ} \mathrm{C}$ for $48-72$ hours. After incubation (Figure 1), the plates were examined through a stereoscopic microscope (Stereoscopic, Nikon, Japan) under reflected light. The number of colonies was counted and reported based on the colony forming unit (CFU) via the manual colony counter (AMETEK, Inc. and Reichert, Inc., Canada).

\section{Disinfection Protocol}

Based on the manufacturer guideline, we applied $10 \mathrm{~mL}$ ASEPTOPRINT ${ }^{\circledR}$ spray to each of the dentures in closed packs for 60 seconds. Then, the dentures were rinsed cautiously for 4 minutes. Finally, swab sampling was done in the same way as the previous disinfection.

\section{Plasma Treatment}

The laboratory pulsed plasma reactor (Tehran, Iran) was used in this experiment. Cold plasma was generated by high voltage discharge in a vacuum chamber (diameter $250 \mathrm{~mm}$, height $500 \mathrm{~mm}$, vacuum100 Pa) with a dielectric barrier discharge (DBD) mode and between the electrodes of the discharge condenser located on a high voltage table, where the sample was placed in glow position. With a relatively high gas flow that varied with the plasma chamber size, the plasma discharge formed
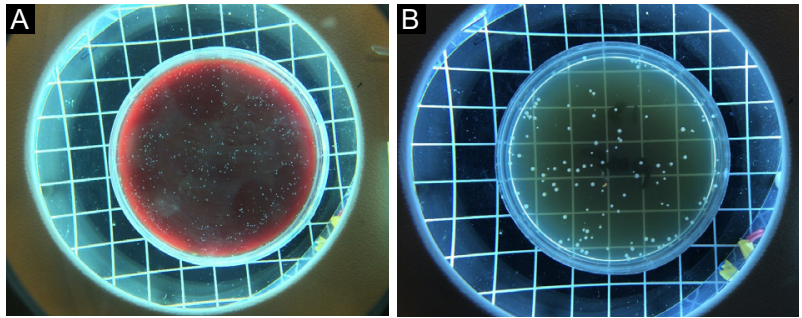

Figure 1. (A) Colonies on blood agar exhibit a whitish and rough surface appearance, which resembled the characteristics of Streptococcus mutans colonies. (B) The appearance of colonies cultivated on Sabouraud Dextrose Agar (SDA) showed yellow-white colonies with some extensions to the surroundings which mimic Candida albicans colonies (Observed under the Manual Colony Counter, AMETEK, Inc. and Reichert, Inc., Canada).

Table 1. Information Provided by the ASEPTOPRINT ${ }^{\circledR}$ Spray Manufacturer

\begin{tabular}{|c|c|c|c|}
\hline Commercial Name & Components & Exposure time & Physical and Chemical Properties \\
\hline $\begin{array}{l}\text { ASEPTOPRINT® Spray CH- } \\
071046\end{array}$ & $\begin{array}{l}20 \mathrm{~g} \text { ethanol, } 28 \mathrm{~g} \text { 1-propanol, } 0.056 \mathrm{~g} \\
\text { quaternary ammonium compounds }\end{array}$ & 60 seconds & $\begin{array}{l}\text { Physical state: Clear, non-viscous liquid } \\
\text { Colour: Colourless } \\
\text { Scent: Of alcohol, lemon } \\
\text { pH-value: Neutral } \\
\text { Shelf Life: } 48 \text { months }\end{array}$ \\
\hline
\end{tabular}


inside the chamber could be blown out of the chamber to form a brush-shaped low-temperature plasma jet, such as a low-temperature atmospheric plasma brush (Figure 2). This plasma source could operate under very low electrical power $(30 \mathrm{~W})$ and as a result, very low plasma temperature could be achieved. A special mesh was designed and used in order to standardize the amount of cold plasma radiation all around the dentures (Figure 2).

Data Analysis

Finally, all data were analyzed using SPSS 16 (SPSS Inc., Chicago, IL, USA). Data were analyzed by conducting a one-way analysis of variance between the groups at each point of time and using the repeated-measures ANOVA for comparing within the experimental groups. Statistical significance was defined at $P<0.05$.

\section{Results}

Microbial isolation prior to the disinfection of all 30 specimens on blood agar and SDA showed a high mean of CFU without any significant difference $(P>0.05)$ (Tables 2 and 3 ). The differences between groups before and after disinfection were significant within all three experimental groups. After disinfection via ASEPTOPRINT ${ }^{\circledR}$ Spray or cold plasma radiation (30/60 seconds), cold plasma treatment for 30 seconds represented the least antimicrobial activity and the highest CFU on both blood agar and SDA cultures. Comparisons between the groups indicated a statistically significant reduction in the number of colonies among cold plasma treatment groups (30 or 60 seconds) and ASEPTOPRINT ${ }^{\circledR}$ spray disinfection $(P<0.05)$. Cold plasma treatments $(30$ and 60 seconds) more significantly decreased the number of colony-forming units in the SDA culture compared to the colonies of microbes which grew on blood agar (2.01 CFU vs. $1.56 \mathrm{CFU}$ and $2.48 \mathrm{CFU}$ vs. $1.71 \mathrm{CFU})$.

\section{Discussion}

In the current study, we aimed to compare the efficacy

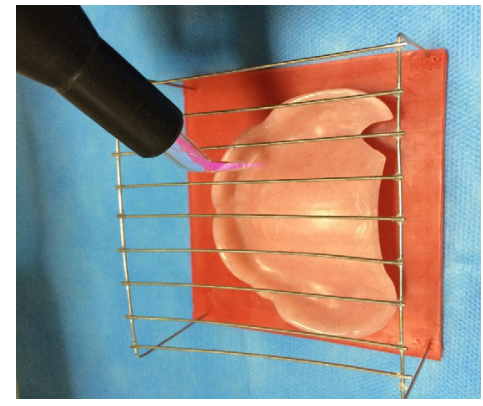

Figure 2. The specially designed mesh was used in order to standardize the amount of cold plasma radiation all around the dentures.

of a newly proposed method for disinfection (cold plasma treatment) with a routinely used alcoholbased disinfectant for the disinfection of the acrylicbased dentures. According to our findings, one of the advantages of this novel technique could be its ability to reduce Candida albicans as the main pathogen for denture stomatitis. Denture stomatitis is a frequent oral disease among geriatric patients, which mainly occurs under the host-related conditions and the imbalance of oral microflora originating from local factors such as denture wearing. The focus in the treatment of denture stomatitis is to eliminate the source of the pathogens which have been colonized in the contaminated dentures. ${ }^{22}$ Therefore, the utilization of a safe protocol for disinfection without any harmful effect on the physical properties of the acrylicbased dentures is essential. ${ }^{23,24}$

In this study, it was hypothesized that cold plasma treatment could be used as an alternative method for the disinfection of the acrylic-based dentures instead of the routinely used ASEPTOPRINT ${ }^{\circledR}$ spray solution as a chemical disinfectant. Our results represented that cold plasma treatment could decrease the amount of contamination in 30 and 60 seconds. However, ASEPTOPRINT $^{\circledR}$ spray was a more effective and reliable technique of disinfection compared to cold plasma treatment.

Table 2. The Mean Colony-Forming Units (CFU) on Blood Agar Culture at 2 Points of Time

\begin{tabular}{lcc}
\hline \multirow{2}{*}{ Experimental Groups } & Before Disinfection & After Disinfection \\
\cline { 2 - 3 } & Mean $($ CFU) \pm Standard Deviation & Mean $($ CFU) \pm Standard Deviation \\
\hline ASEPTOPRINT ${ }^{\circledR}$ Spray (OCC, Switzerland) & $5.27 \pm 1.21$ & $0.95 \pm 1.23$ \\
Plasma 30 seconds & $5.28 \pm 0.66$ & $3.72 \pm 0.38$ \\
Plasma 60 seconds & $5.13 \pm 0.25$ & $3.42 \pm 0.78$ \\
\hline
\end{tabular}

Table 3. The Mean Colony-Forming Units (CFU) on Sabouraud Dextrose Agar (SDA) Culture at 2 Points of Time

\begin{tabular}{lcc}
\hline \multirow{2}{*}{ Experimental Groups } & Before Disinfection & After Disinfection \\
\cline { 2 - 3 } & Mean $($ CFU) \pm Standard Deviation & Mean (CFU) \pm Standard Deviation \\
\hline ASEPTOPRINT ${ }^{\circledR}$ Spray (OCC, Switzerland) & $4.95 \pm 0.99$ & $0.77 \pm 1.25$ \\
Plasma 30 seconds & $4.61 \pm 0.69$ & $2.01 \pm 1.53$ \\
Plasma 60 seconds & $5.15 \pm 0.28$ & $2.67 \pm 1.10$ \\
\hline
\end{tabular}


According to a high microbial load of an oral cavity, blood agar and SDA culture were used to cover nearly most of the potentially wide variety of microbes including bacteria and fungi. It has been indicated that the application of cold plasma can have some antimicrobial impacts. Goree et $\mathrm{a}^{25}$ showed that the 10-second application of a plasma needle could eliminate Streptococcus mutans in oral biofilms. In 2011, Yang et $\mathrm{al}^{26}$ fabricated a novel toothbrush which irradiated cold argon plasma. They represented that this treatment could eliminate streptococcus mutans and lactobacillus in the collected saliva. Moreover, they indicated that 11-15 seconds of cold plasma therapy was required for the inactivation of streptococcus mutans. Nevertheless, lactobacillus needed more time to be thoroughly eliminated. Although the number of microorganisms was reduced in our investigation, the amount of reduction was not significant compared to the conventional method of disinfection (chemical solutions). Zamperini et $\mathrm{al}^{24}$ showed that the application of $\mathrm{ArO} 2$ cold plasma with 70-watt power for 5 minutes could significantly decrease the amount of cultivated Candida albicans on artificial acrylic resin blocks. This fact can be explained by our restricted time of exposure and its physical properties.

In addition, it has been represented that cold plasma therapy can decrease the number of intra-root canal microorganisms. Jiang et $\mathrm{al}^{27}$ utilized a newly designed device that irradiated cold plasma into dental root canals. Although they reported a very high antibacterial impact of this method, they concluded that the technique was highly dependent on the direct radiation. Thus, one of the possible explanations for our data is the insufficient radiation which covers the complete surface of the dentures. It seems that the utilization of a larger device may increase the effectivity due to the maintenance of appropriate, complete coverage for direct irradiation.

In conclusion, our results represented that the antimicrobial effect of ASEPTOPRINT ${ }^{\circledast}$ spray solution on both microbial and fungal microorganisms was more significant than cold plasma application. The time of plasma application did not show any significant differences in this study. Further studies are required to unravel the role of cold plasma radiation in disinfection.

\section{Ethical Considerations}

Ethical approval was obtained from the 14th research ethics committee (2016-01-15) of Shahid Beheshti Medical University.

\section{Conflict of Interests}

The authors declare no conflict of interest.

\section{Acknowledgment}

This research was supported by the grant of Laser Application in Medical Sciences Research Center of Shahid Beheshti Medical University.

\section{References}

1. Gupta S, Rani S, Garg S. Infection control knowledge and practice: A cross-sectional survey on dental laboratories in dental institutes of North India. J Indian Prosthodont Soc. 2017;17:348-54. doi: 10.4103/jips.jips_5_17

2. Bhat VS, Shetty MS, Shenoy KK. Infection control in the prosthodontic laboratory. J Indian Prosthodont Soc. 2007;7(2):62-5. doi: 10.4103/0972-4052.33997

3. Kumari KV, Subhash A. Infection Control in Dental Clinic and Laboratory. Indian Journal of Mednodent and Allied Sciences. 2014;2(2). doi: 10.5958/2347-6206.2014.00009.0

4. Williams DW, Chamary N, Lewis MA, Milward PJ, McAndrew R. Microbial contamination of removable prosthodontic appliances from laboratories and impact of clinical storage. Br Dent J. 2011;211(4):163-6. doi: 10.1038/ sj.bdj.2011.675

5. Al Mortadi N, Al-Khatib A, Alzoubi KH, Khabour OF. Disinfection of dental impressions: knowledge and practice among dental technicians. Clin Cosmet Investig Dent. 2019;11:103-8. doi: 10.2147/CCIDE.S205144

6. Centers for Disease Control and Prevention. Summary of Infection Prevention Practices in Dental Settings: Basic Expectations for Safe Care. Atlanta, GA: Centers for Disease Control and Prevention, US Dept of Health and Human Services; 2016.

7. British Dental Association. Health Technical Memorandum 01-05: Decontamination in Primary Care Dental Practices. London: Department of Health; 2013.

8. ADA Council on Scientific Affairs and ADA Council on Dental Practice. Infection control recommendations for the dental office and the dental laboratory. J Am Dent Assoc. 1996; 127:672-80.

9. Carvalho CF, Vanderlei AD, Marocho SM, Pereira SM, Nogueira L, Paes-Junior TJ. Effect of disinfectant solutions on a denture base acrylic resin. Acta Odontol Latinoam. 2012;25(3):255-60.

10. Aslanimehr M, Mojarad N, Ranjbar S, Aalaei S. In vitro comparison of the effects of microwave irradiation and chemical and mechanical methods on the disinfection of complete dentures contaminated with Candida albicans. Dent Res J (Isfahan). 2018;15(5):340-6. doi: 10.4103/17353327.240477

11. Pavarina AC, Pizzolitto AC, Machado AL, Vergani CE, Giampaolo ET. An infection control protocol: effectiveness of immersion solutions to reduce the microbial growth on dental prostheses. J Oral Rehabil. 2003;30(5):532-6. doi: 10.1046/j.1365-2842.2003.01093.x

12. Orsi IA, Andrade VG. Effect of chemical disinfectants on the transverse strength of heat-polymerized acrylic resins submitted to mechanical and chemical polishing. J Prosthet Dent. 2004;92(4):382-8. doi: 10.1016/j. prosdent.2004.07.015

13. Rutala WA, Weber DJ. Selection of the ideal disinfectant. Infect Control Hosp Epidemiol. 2014;35(7):855-65. doi: $10.1086 / 676877$

14. Pavarina AC, Vergani CE, Machado AL, Giampaolo ET, Teraoka MT. The effect of disinfectant solutions on the hardness of acrylic resin denture teeth. J Oral Rehabil. 2003;30(7):749-52. doi: 10.1046/j.1365-2842.2003.01145.x 15. Machado AL, Giampaolo ET, Vergani CE, Souza JF, 
Jorge $\mathrm{JH}$. Changes in roughness of denture base and reline materials by chemical disinfection or microwave irradiation: surface roughness of denture base and reline materials. J Appl Oral Sci. 2011;19(5):521-8. doi: 10.1590/ s1678-77572011000500015

16. Sagripanti JL, Bonifacino A. Cytotoxicity of liquid disinfectants. Surg Infect (Larchmt). 2000;1(1):3-14. doi: $10.1089 / 109629600321245$

17. Ziuzina D, Boehm D, Patil S, Cullen P, Bourke P. Cold plasma inactivation of bacterial biofilms and reduction of quorum sensing regulated virulence factors. PloS One. 2015;10(9):e0138209. doi: 10.1371/journal.pone.0138209

18. Kim B, Yun H, Jung S, Jung $Y$, Jung $H$, Choe W, et al. Effect of atmospheric pressure plasma on inactivation of pathogens inoculated onto bacon using two different gas compositions. Food Microbiol. 2011;28(1):9-13. doi: 10.1016/j.fm.2010.07.022

19. Song HP, Kim B, Choe JH, Jung S, Moon SY, Choe W, et al. Evaluation of atmospheric pressure plasma to improve the safety of sliced cheese and ham inoculated by 3-strain cocktail Listeria monocytogenes. Food Microbiol. 2009;26(4):432-6. doi: 10.1016/j.fm.2009.02.010

20. Chen YJ, Chen YC, Chung WC, Tong BS, Chang MB. Evaluation of the effectiveness of nonthermal plasma disinfection. Environ Technol. 2019:In Press. doi: 10.1080/09593330.2019.1583289

21. Ulbin-Figlewicz N, Jarmoluk A, Marycz K. Antimicrobial activity of low-pressure plasma treatment against selected foodborne bacteria and meat microbiota. Ann Microbiol.
2015;65(3):1537-46. doi: 10.1007/s13213-014-0992-y

22. Borges AC, Lima GMG, Nishime TMC, Gontijo AVL, Kostov KG, Koga-Ito CY. Amplitude-modulated cold atmospheric pressure plasma jet for treatment of oral candidiasis: In vivo study. PLoS One. 2018;13(6):e0199832. doi: 10.1371/journal.pone.0199832

23. Moisan M, Barbeau J, Crevier M-C, Pelletier J, Philip N, Saoudi B. Plasma sterilization. Methods and mechanisms. Pure Appl Chem. 2002;74(3):349-58. doi: 10.1351/ pac200274030349

24. Zamperini CA, Machado AL, Vergani CE, Pavarina AC, Giampaolo ET, da Cruz NC. Adherence in vitro of Candida albicans to plasma treated acrylic resin. Effect of plasma parameters, surface roughness and salivary pellicle. Arch Oral Biol. 2010;55(10):763-70. doi: 10.1016/j. archoralbio.2010.06.015

25. Goree J, Liu B, Drake D, Stoffels E. Killing of S. mutans bacteria using a plasma needle at atmospheric pressure. IEEE Trans Plasma Sci. 2006;34(4):1317-24. doi: 10.1109/ TPS.2006.878431

26. Yang B, Chen J, Yu Q, Li H, Lin M, Mustapha A, et al. Oral bacterial deactivation using a low-temperature atmospheric argon plasma brush. Journal of dentistry. J Dent. 2011;39(1):48-56. doi: 10.1016/j.jdent.2010.10.002

27. Jiang C, Chen MT, Gorur A, Schaudinn C, Jaramillo DE, Costerton JW, et al. Nanosecond pulsed plasma dental probe. Plasma Process Polym. 2009;6(8):479-83. doi: 10.1002/ppap.200800133 